\title{
Entrainment enhances theta oscillations and improves episodic memory
}

\author{
Brooke M. Roberts ${ }^{1 *}$, Alex Clarke ${ }^{2,3^{*}}$, Richard J. Addante ${ }^{4}$, Charan Ranganath ${ }^{1,5}$
}

1. Department of Psychology, University of California at Davis, Davis, CA 95616

2. Department of Psychology, University of Cambridge, Cambridge, UK.

3. Department of Psychology, Anglia Ruskin University, Cambridge, UK.

4. Department of Psychology, California State University, San Bernardino, San Bernardino CA 92407

5. Center for Neuroscience, University of California at Davis, Davis, CA 95618

* These authors contributed equally

\section{Corresponding Author:}

Charan Ranganath (cranganath@ucdavis.edu).

Phone: 530-757-8865; Fax: 530-757-8640; Address: 1544 Newton Court, Davis, CA 95618, U.S.A.

Conflict of Interest: The authors declare no competing financial interests.

Acknowledgements: We thank Steve Luck and Mariam Aly for helpful suggestions on previous drafts of this manuscript. This project was supported by a Guggenheim Fellowship and by a Vannevar Bush Fellowship (Office of Naval Research Grant N00014-15-1-0033). Any opinions, findings, and conclusions or recommendations expressed in this material are those of the author(s) and do not necessarily reflect the views of the Office of Naval Research or the U.S. Department of Defense. 


\begin{abstract}
Neural oscillations in the theta band have been linked to episodic memory, but it is unclear whether activity patterns that give rise to theta play a causal role in episodic retrieval. Here, we used rhythmic auditory and visual stimulation to entrain neural oscillations to assess whether theta activity contributes to successful memory retrieval. In two separate experiments, human subjects studied words and were subsequently tested on memory for the words ("item recognition") and context in which each had been previously studied ("source memory"). Between study and test, subjects in the entrainment groups were exposed to audiovisual stimuli designed to enhance activity at $5.5 \mathrm{~Hz}$, whereas subjects in the control groups were exposed to white noise (Expt. 1) or $14 \mathrm{~Hz}$ entrainment (Expt. 2). Theta entrainment selectively increased source memory performance in both studies. Electroencephalography (EEG) data in Expt. 2 revealed that theta entrainment resulted in band-specific enhancement of theta power during the entrainment period and during post-entrainment memory retrieval. These results demonstrate a direct link between theta activity and episodic memory retrieval. Targeted manipulation of theta activity could be a promising new approach to enhance theta activity and memory performance in healthy individuals and in patients with memory disorders.
\end{abstract}




\section{Introduction:}

In the rat hippocampus, theta oscillations temporally structure the activity of neural ensembles during memory retrieval and spatial navigation (Hasselmo et al., 2002), and these oscillations have been linked to successful learning and retention (Landfield et al., 1972; Winson, 1978). Theta activity is also prominent in human EEG recordings in the 4-8 $\mathrm{Hz}$ frequency range, and studies have linked scalp-recorded theta to medial prefrontal (Asada et al., 1999; Ishii et al., 1999) and parietal sources (Foster and Parvizi, 2012; see Hsieh and Ranganath, 2014 for review) that are known to exhibit high functional connectivity with the hippocampus (Kahn et al., 2008; Libby et al., 2012; Wang et al., 2014, 2015; Wang et al., 2016). EEG studies show that theta activity is enhanced during successful memory encoding and retrieval, particularly on contextdependent memory tasks that are thought to depend on hippocampal function (Klimesch et al., 1997; Jacobs et al., 2006; Haenschel et al., 2009; Rutishauser et al., 2010; Addante et al., 2011; Foster et al., 2013; Staudigl and Hanslmayr, 2013; Anderson and Hanslmayr, 2014; Fuentemilla et al., 2014; Hsieh and Ranganath, 2014; Clarke, Roberts and Ranganath, 2018; Crivelli-Decker et al., 2018).

If theta activity contributes to episodic memory retrieval, then direct manipulations of theta activity could significantly affect memory performance. Such evidence would suggest that theta oscillations are not mere epiphenomena (see Yartsev et al., 2011), and moreover, that targeted enhancement of theta activity could be used to improve episodic memory in healthy individuals or in patients with memory disorders.

Here, we tested whether rhythmic presentation of auditory and visual stimuli could be used to stimulate the brain networks that generate theta activity, through a process known as "oscillatory entrainment" (Walter, 1953; Lakatos et al., 2008; Thut et al., 2011; Calderone et al., 2014; Herrmann et al., 2015). In studies of visual processing, entrainment has been shown to enhance oscillatory activity and modulate spiking activity and behavioral performance (Lakatos et al., 2008; Calderone et al., 2014). These findings suggest that that rhythmic auditory and visual stimuli can entrain oscillatory activity, and that processing of stimuli during entrainment can be enhanced if stimuli are delivered at the optimal phase of the ongoing oscillation. Here, we were interested in determining whether entrainment can be used to stimulate brain networks that generate theta oscillations. Specifically, we hypothesized that entrainment of theta oscillations would have lasting effects on episodic memory retrieval and on spontaneous theta activity.

To address this question, we conducted two experiments in which participants were exposed to audiovisual entrainment (AVE) at theta frequency $(5.5 \mathrm{~Hz}$ ) between the study (encoding) and test (retrieval) phases of an episodic memory paradigm. In a study using a similar memory paradigm, we (Addante et al., 2011) found that frontal and parietal theta oscillations were enhanced during successful retrieval of contextual information associated with learned items ("source memory"), but no effects were evident during successful recognition of items in the absence of correct source memory retrieval ("item memory"). These results led us to predict 
that, if AVE has lasting effects on networks that generate theta oscillations, then we would expect to see improved source memory performance and enhanced theta activity during source memory retrieval.

\section{Materials and Methods:}

\section{Participants:}

Experiment 1: 50 right-handed subjects (26 females) ages 18 - 25 (average $20.3 \pm 0.31$ years) were recruited from the UC Davis undergraduate community. 25 subjects each were assigned to the theta $(5.5 \mathrm{~Hz}$ ) entrainment group and to the control (white noise) condition. At the point of data analysis, 5 subjects were removed due to chance source memory discriminability. Chance discriminability was defined as Percent Source Correct - Percent Source Incorrect $\leq 1 \%$. The final analysis for the theta entrainment group included 24 subjects, and 21 subjects were included in the final analysis for the control group. However, inclusion of these subjects would not change the results--all of the major findings were statistically significant even when the entire sample was included.

Experiment 2: 40 right-handed subjects (22 females) ages 18-26 (average $20.5 \pm 0.27$ SEM) were recruited from the UC Davis undergraduate community. 20 subjects each were assigned to the $5.5 \mathrm{~Hz}$ entrainment group and the $14 \mathrm{~Hz}$ entrainment control group.

Both experiments were approved by the Institutional Review Board at the University of California, Davis. Written informed consent was obtained from each subject for both experiments before participation.

\section{Task:}

During the study phase (i.e., "encoding blocks"), subjects incidentally learned 200 words in the context of two different incidental encoding tasks. Words were studied in 4 blocks, with each block consisting of 50 encoding trials. During two study blocks, subjects indicated whether each word corresponded to a living thing (for the "alive" task), and during two blocks, they indicated whether it corresponded to something that is man-made (for the "man-made" task). The order of "alive" and "manmade" blocks was counterbalanced across subjects. Following the study blocks, subjects received 36 minutes of either theta entrainment at $5.5 \mathrm{~Hz}$, or control (white noise in Study 1 and $14 \mathrm{~Hz}$ entrainment in Study 2).

At test (i.e. retrieval), stimuli presented during the encoding phase were randomly intermixed with an additional 100 new words (lures), for a total of 300 test stimuli. Retrieval was divided into 6 testing blocks, each consisting of 50 words. Each trial commenced with presentation of a fixation cross in the center of the screen for $1000 \mathrm{~ms}$, followed by central presentation of a word for $1500 \mathrm{~ms}$. Next, participants were prompted to make self-paced item and source decisions (see Fig. 1). For item-recognition judgments, subjects responded using a 5-point confidence scale, with 5 indicating that they were sure it was studied, 4 indicating that it was probably studied, 3 indicating guessing, 2 indicating it was probably new, and 1 indicating that they were 
sure it was new. For source decisions, subjects again used a 5-point confidence scale, with 5 indicating that they were sure the word was presented in the man-made task, 4 indicating it was probably from the man-made task, 3 indicating guessing, 2 indicating it was probably from the alive task, and 1 indicating that they were sure it was from the alive task. During source judgments subjects were instructed to respond with 3 for new items. Studied items that received 5 or 4 responses for item judgments were treated as item recognition "hits". Source memory hits corresponded to items studied in the alive task that received 1 or 2 responses and items studied in the man-made task that received 4 or 5 responses. Percentages were calculated based on the total number of trials, excluding missed trials in which subjects failed to make a response, and trials in which subjects made a 3 response (guesses). Item Only responses included trials with correct item hits that were not associated with correct source hits.

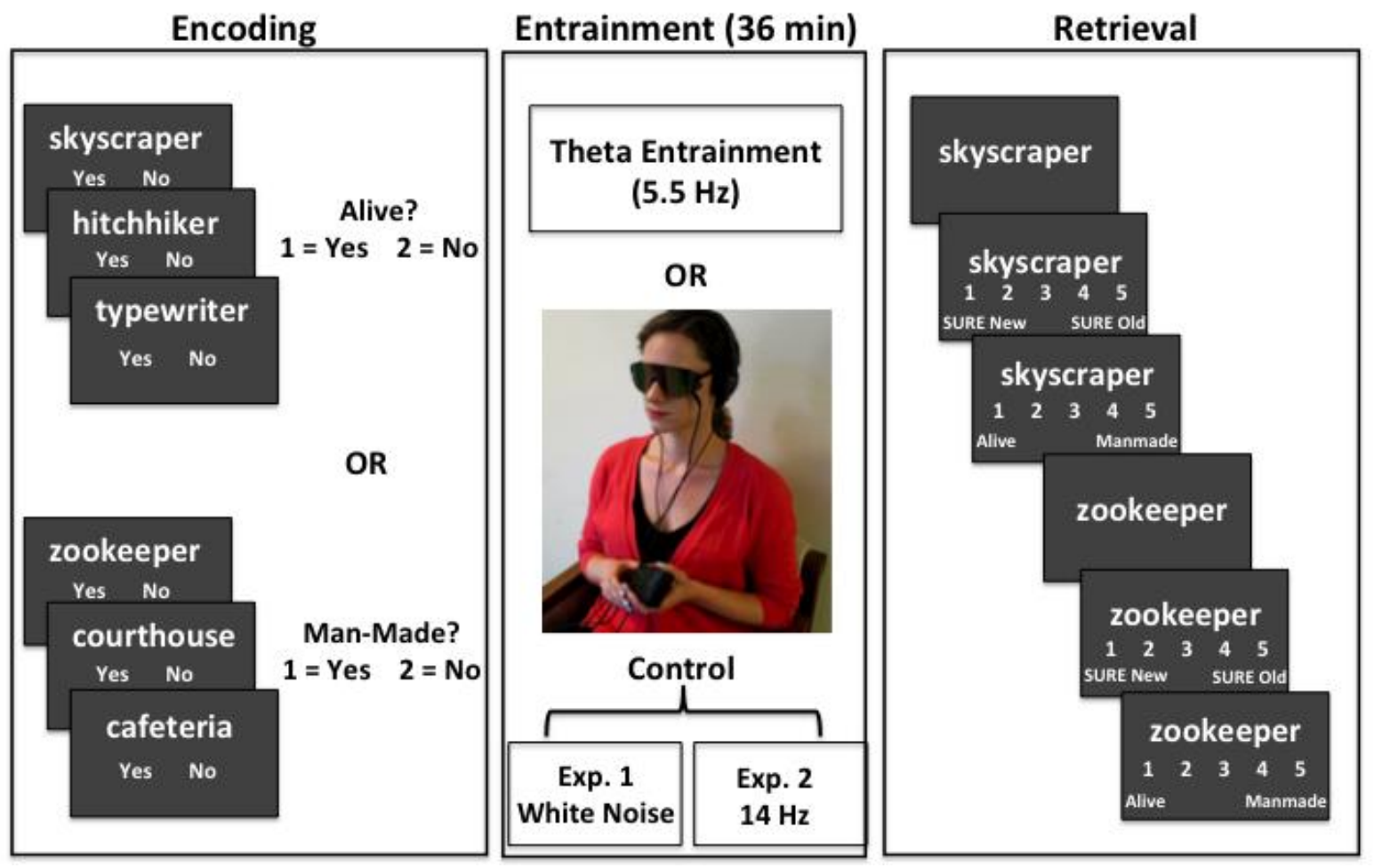

Figure 1: Schematic of the episodic memory task. Participants incidentally learned lists of visually-presented words while performing two semantic decision tasks. During "alive" blocks, they indicated whether each word represented a living thing, and during "man-made" blocks, they indicated whether it represented something that was man-made. Following 36 minutes of theta entrainment or a control condition, subjects completed a surprise memory test that required-item and source recognition confidence judgments. In Expt. 2, EEG was recorded during entrainment and during memory retrieval. 


\section{AVE procedure:}

Between encoding and retrieval phases subjects received 36 minutes of either theta entrainment at $5.5 \mathrm{~Hz}$, or control (white noise in Study 1 and $14 \mathrm{~Hz}$ entrainment in Study 2). Subjects were presented with lights and sounds targeting a frequency of $5.5 \mathrm{~Hz}$, or control, in order to drive theta oscillations prior to memory retrieval. A commercially available device (DAVID PAL 36 by Mind Alive, Inc., Edmonton, Canada) was used to administer visual stimulation (via LED lights embedded on the inner edge of darkened eyeglasses) and isochronic pulsed tones (via headphones) targeting $5.5 \mathrm{~Hz}$ (theta entrainment groups) or $14 \mathrm{~Hz}$ (control group for Experiment 2) for 36 minutes. The protocols, and duration, are pre-set in the device which delivers synchronous, in phase, audio-visual stimulation. For the control condition in Experiment 1 , subjects listened to white noise for 36 minutes using the same headphones. Subjects also wore the eyeglasses with the lights turned off. All subjects were instructed to attend to the lights and sounds, and to relax during entrainment, but not to fall asleep. Subjects could close their eyes or leave them slightly open to ensure the comfort of each participant during the entrainment period, as the lights remain visible with eyes closed.

\section{EEG Recordings:}

In Experiment 2, EEG was recorded at a sampling rate of $1024 \mathrm{~Hz}$ with respect to a common mode sense (CMS) active electrode located on the scalp near electrode site $\mathrm{Cz}$ during the entrainment period and subsequent memory test using a BioSemi (http://www.biosemi.com) Active Two system. EEG recordings were taken from 64 active $\mathrm{Ag} / \mathrm{AgCl}$ scalp electrodes embedded in an elastic cap, with electrode locations corresponding to an extended version of the international 10/20 system (Klem et al., 1999). Recordings were also taken from active electrodes placed on the left and right mastoids, and horizontal and vertical electroculogram (EOG) recordings were made from electrodes placed lateral to each eye, and above and below the left eye. The skin under the electrodes was slightly abraded using a blunt applicator needle, and electrodes were filled with electrolyte gel. Testing was conducted in a sound-attenuated testing chamber. Subjects were instructed to minimize muscle tension, eye movements and blinking during, particularly during the fixation periods and test stimulus presentation. Following the response period for each trial a slide was inserted on the screen indicating that it was "OK to Blink." Participants were encouraged restrict any blinks or eye movements to this period. This time period was not included in EEG analysis.

\section{EEG data analysis:}

\section{Power estimates during entrainment:}

The EEGLAB toolbox (Delorme and Makeig, 2004) in Matlab was used in EEG data analysis. Examination of the spectral characteristics of the AVE signals revealed that, stimulation frequencies fluctuated in the first 10-15 minutes, whereas stimulation was focused on the targeted frequency during the 15-30 minutes following the onset of AVE. Accordingly, analyses of EEG data during entrainment focused on this epoch, when AVE was specifically focused on the targeted frequency. 
Our analyses of EEG activity during AVE focused on effects on oscillatory power. We were primarily interested in testing the hypothesis that AVE stimulation would enhance EEG power at the targeted entrainment frequency. The AVE device used in this study was not equipped to generate precise records of stimulus onsets that could be synchronized to the EEG recordings, so we could not determine the phase relationship between the AVE stimuli and the evoked EEG signal. Although the phase relationship between AVE and EEG activity might be relevant for understanding effects of entrainment on online processing, in the present study, we were interested in the effects of AVE on behavior and activity during the post-entrainment period. Accordingly, the phase of EEG evoked during AVE was not relevant to our experimental questions.

EEG data were resampled to $1000 \mathrm{~Hz}$ and filtered between 1 and $55 \mathrm{~Hz}$ using a Blackman windowed sinc FIR filter (transition bandwidth $0.2 \mathrm{~Hz}$, length 27500 points). Data were segmented into 2 second consecutive, non-overlapping epochs and each epoch was de-meaned. Bad channels were detected by manual inspection of the data, and were removed before data were re-referenced to the average of the left and right mastoids. Independent component analysis (ICA) was run using runica (Delorme et al., 2007; Jung et al., 2000a,b), and SASICA (Chaumon et al., 2015) was used for the detection of artifactual components to reject, which were visually inspected for validity. The power spectra were calculated using Bartlett's method which averages together the non-overlapping periodograms given by each epoch. Specifically, spectral power was calculated for each epoch using a fast Fourier transform (FFT) for frequencies between 1 and $20 \mathrm{~Hz}$, in $0.5 \mathrm{~Hz}$ steps, normalized using a log transform and averaged over epochs.

\section{Time-frequency analysis during memory retrieval:}

Data analyses were performed offline using the EEGLab Toolbox (Delorme and Makeig, 2004) and Fieldtrip Toolbox (Oostenveld et al., 2011). Analysis during memory retrieval focused on trials with correct item recognition only, trials with correct item recognition and source memory performance, and correct rejections. All other trials were excluded from analysis. EEG data were down-sampled to $512 \mathrm{~Hz}$, re-referenced to the average of the left and right mastoids, and highpass filtered at $0.5 \mathrm{~Hz}$. Data were segmented into 3.65s epochs following starting $1.25 \mathrm{~s}$ prior to the onset of the word, and extending to $2.4 \mathrm{~s}$ following the presentation of the word for each trial in order to encompass the baseline period, in which subjects focused on a fixation cross in the center of the screen through the test stimulus presentation and response periods. These epochs were visually inspected to reject any trials that appeared to contain excessive noise. Independent component analysis (ICA) was used to correct for artifacts in the remaining trials (Delorme et al., 2007; Jung et al., 2000a,b). Epochs were visually inspected again following ICA to reject any remaining trails that appeared to contain residual EOG, EMG, skin potential, or other artifacts. Following artifact rejection, the remaining trials were included in time-frequency analysis. 
Time-frequency analysis was performed using 5.7 cycle Morlet wavelet decomposition (Roach and Mathalon, 2008) ranging from 2 to $50 \mathrm{~Hz}$ at 47 logarithmically spaced frequencies. The decomposition was performed on epochs for each trial included in analysis. Time-frequency analysis was performed from $450 \mathrm{~ms}$ prior to the test stimulus presentation to $1600 \mathrm{~ms}$ following the presentation of the test stimulus. Data were baseline corrected to the fixation period prior to test stimulus presentation (-450 to $0 \mathrm{~ms})$.

After Morlet wavelet decomposition of each trial, oscillatory power was averaged for correct item recognition only trials and correct item recognition and source memory trials for each subject in both the $5.5 \mathrm{~Hz}$ and $14 \mathrm{~Hz}$ entrainment groups, resulting in condition specific timefrequency representations.

\section{Statistical analysis:}

Statistical analyses were performed with Fieldtrip (Oostenveld, Fries, Maris, \& Schoffelen, 2011) on frequencies of interest. For the analysis of the entrainment period, the data were analyzed at $5.5 \mathrm{~Hz}$ and at $14 \mathrm{~Hz}$. For the retrieval data, theta activity $(4-6 \mathrm{~Hz})$ and low beta activity $(12-16 \mathrm{~Hz})$ were analyzed. Differences between the entrainment and control groups were tested using a cluster-based permutation approach to identify significant electrode clusters within the frequency band (Maris and Oostenveld, 2007). Differences between conditions were established using a two-tailed independent t-test with an alpha of 0.05 , and the sum of cluster-forming tvalues were calculated based on neighboring electrodes (with a minimum of 2 significant electrodes forming a connection). Group assignments were randomly shuffled and the maximum cluster mass was retained from the permuted data. The data were permuted 5000 times to create a permutation distribution that was used to calculate significance values for observed clusters in the original data.

For the EEG data during entrainment, we tested for topographic effects, and for the retrieval EEG data we tested for topographic effects over time, between 0.25 seconds and 1 second. The time period from 0 to 0.25 seconds was not analyzed due to possible influences of pre-stimulus activity on wavelet power estimates.

\section{Results:}

Overview:

Two experiments were conducted to assess effects of entrainment (see Fig. 1). Experiment 1 was a behavioral study, in which item and source recognition was compared between participants exposed to $5.5 \mathrm{~Hz}$ entrainment and participants who were exposed to white noise. Experiment 2 used the same design, except that participants undergoing $5.5 \mathrm{~Hz}$ entrainment were compared against a $14 \mathrm{~Hz}$ entrainment control group, and EEG data were collected during entrainment and recognition.

\section{Experiment 1:}


As noted earlier, theta activity is enhanced during source memory retrieval tasks that are thought to depend on the hippocampus. We predicted that AVE would stimulate the cortical networks that generate theta, thereby increasing the likelihood of theta states and enhancing source memory performance during the retrieval test. Consistent with this prediction, results from Experiment 1 (Fig. $2 \mathrm{~A}$ and Table 1) showed that the proportion of trials associated with correct Item and Source memory judgments ("Item+Source") was significantly higher for the entrainment group compared to the control (white noise) group ( $F[1,43]=4.30 ; p=0.044$; Cohen's $d=0.628$ ). There were no significant differences between the entrainment and control groups in the proportion of trials for correct "Item Only" trials $(F[1,43]=1.26 ; p=0.725)$. The rate of false alarms for item recognition judgments also did not significantly vary between theta entrainment and control groups $(F[1,43]=0.474 ; p=0.495)$. Consistent with these findings, signal-detection-based analyses revealed that no significant between-group differences in item recognition discriminability (i.e. $d^{\prime} ; F[1,43]=2.22 ; p=0.14$ ), or response bias $(F[1,43]=0.46 ; p$ $=0.50)$. These results indicate that theta entrainment increased the likelihood of successful source memory retrieval, consistent with previous findings demonstrating a specific relationship between theta activity and source memory retrieval (Addante et al., 2011).

A

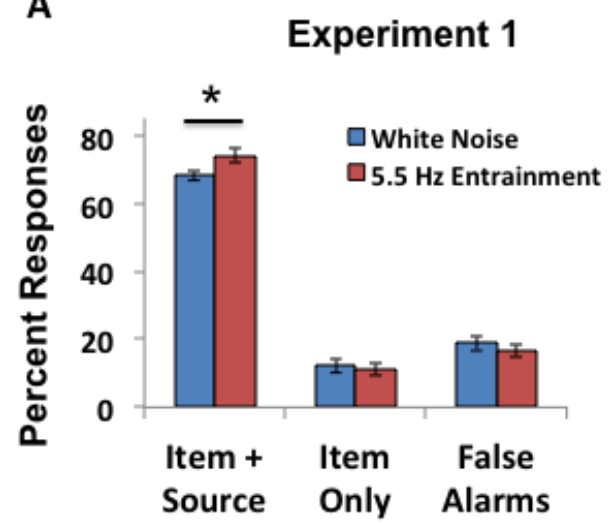

B

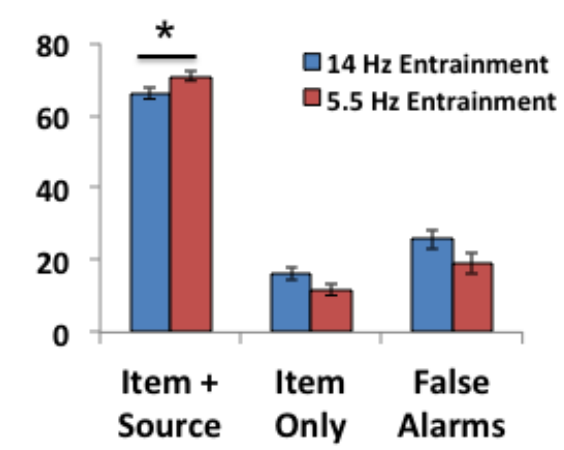

Figure 2: Theta entrainment enhances source memory performance. Proportions of Item + Source hits, Item Only hits, and False Alarms are plotted for (A) Experiment 1 and (B) Experiment 2. Asterisks indicate significant differences between theta entrainment and control groups.

According to dual-process models of recognition memory, Item-Only responses are primarily supported by familiarity, whereas Item+Source responses reflect familiarity and recollection (see Yonelinas, 2002; Mickes et al., 2009). If one assumes that recollection and familiarity are independent, Item-Only hit rates underestimate the effect of familiarity, because they do not take into account the fact that familiarity cannot be estimated for Item+Source hits (see Yonelinas \& Jacoby, 2002). Adapting the equation from Yonelinas \& Jacoby (2002), we estimated familiarity as follows: $p$ (Familiarity) $=p($ ItemOnly $) /[1-p($ Item+Source $)]$. Consistent with our analyses of Item-Only hit rates, this analysis also did not reveal significant effects of entrainment 
on familiarity (Entrainment: $M=0.91 ; S E M=0.023$; Control: $M=0.88$; $S E M=0.044$ : $F[1,43]=0.320$; $p=0.575)$.

\section{Experiment 2:}

In Experiment 2, we sought to: (1) replicate the effects of theta entrainment observed in the first experiment, (2) examine theta activity during entrainment and during the subsequent memory test, and (3) eliminate the possibility that between-group differences were driven by factors related to the white noise control condition, rather than entrainment. The design of Experiment 2 was identical to experiment 1, except that participants in the control group were exposed to AVE at $14 \mathrm{~Hz}$, and EEG was concurrently recorded in order to assess oscillatory activity during entrainment and during the subsequent retrieval phase.

Behavioral results for Experiment 2, summarized in Fig. 2B and Table 1, replicated those of Experiment 1, showing that the proportion of Item + Source trials was significantly higher for the $5.5 \mathrm{~Hz}$ entrainment group compared to the $14 \mathrm{~Hz}$ entrainment group $(F[1,38]=5.02 ; p=0.031$; Cohen's $d=0.709$ ). Similar to experiment 1 , the proportion of correct Item Only hits were not significantly different across the theta entrainment and control groups $(F[1,38]=2.93 ; p=$ 0.095). The rate of false alarms for new items also did not significantly differ across theta entrainment and control groups $(F[1,38]=2.61 ; p=0.114)$. Moreover, signal-detection analyses revealed no difference between entrainment groups in item recognition discriminability $\left(d^{\prime}\right.$; $[F$ $[1.38]=3.28 ; p=0.08]$ or response bias $[F[1,38]=0.01 ; p=0.92]$. Analyses assuming independent familiarity and recollection processes also revealed no significant effects of entrainment group on item familiarity estimates (Entrainment: $M=0.94 ; S E M=0.027$; Control: $M=0.95 ; S E M=0.057 ; F[1,38]=0.0006 ; p=0.981$ ). The striking parallels between the behavioral results of Experiments 1 and 2 demonstrate the robustness of entrainment effects on memory. Furthermore, because each study used different rigorous controls, findings from the two experiments suggest that the between-group differences were driven by theta entrainment and not by factors peculiar to the control condition.

We next analyzed the EEG data in order to determine the direct effects of AVE on oscillatory activity. During entrainment, EEG power was enhanced specifically at the entrainment frequencies for both the $5.5 \mathrm{~Hz}$ entrainment group, and the $14 \mathrm{~Hz}$ control entrainment group (Fig. 3A). Statistical analyses for power at $5.5 \mathrm{~Hz}$ revealed a significant enhancement for the 5.5 $\mathrm{Hz}$ entrainment group compared to the $14 \mathrm{~Hz}$ control group across posterior electrodes (cluster $\mathrm{p}=0.0064$; Figure $3 \mathrm{~B}$ ), while power at $14 \mathrm{~Hz}$ was significantly enhanced for the $14 \mathrm{~Hz}$ group compared to the $5.5 \mathrm{~Hz}$ group across most electrodes, peaking over central electrodes (cluster $\mathrm{p}$ $=0.0036$; Figure 3C). 

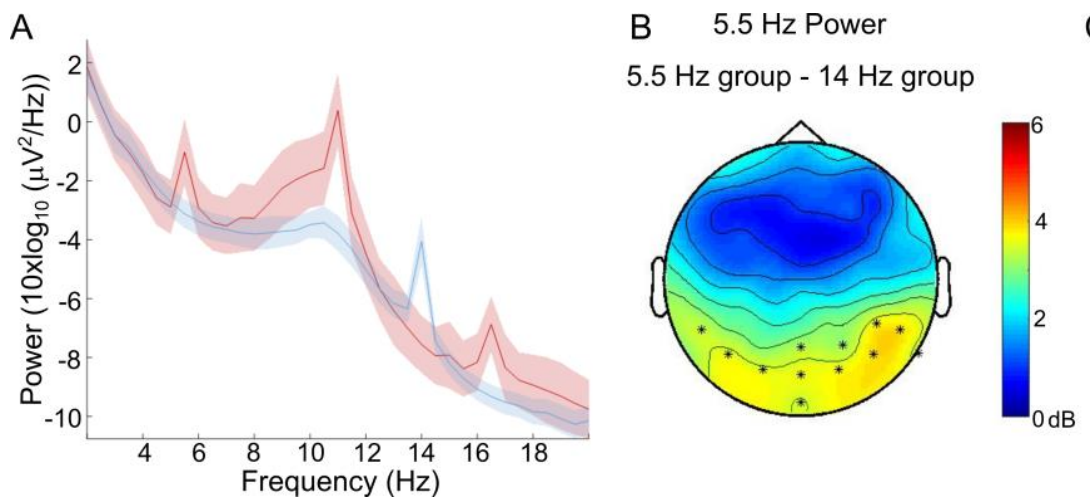

C $\quad 14 \mathrm{~Hz}$ Power

$5.5 \mathrm{~Hz}$ group - $14 \mathrm{~Hz}$ group

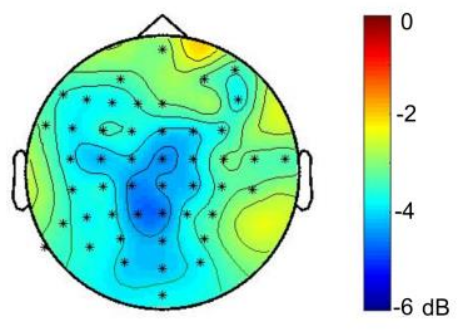

Figure 3: Entrainment enhances EEG power at the stimulation frequencies. A) Power spectrum (averaged across all electrodes) showing peaks for the $5.5 \mathrm{~Hz}$ and $14 \mathrm{~Hz}$ groups at the specific frequencies used during AVE, and effects at the harmonics. B) Topographic map showing enhanced power at $5.5 \mathrm{~Hz}$ for the $5.5 \mathrm{~Hz}$ entrainment group compared to the $14 \mathrm{~Hz}$ entrainment group during AVE. Asterisks indicate the electrodes showing a significant power enhancement for the $5.5 \mathrm{~Hz}$ group compared to the $14 \mathrm{~Hz}$ group. C) Topographic map showing enhanced power at $14 \mathrm{~Hz}$ for the $14 \mathrm{~Hz}$ control group compared to the $5.5 \mathrm{~Hz}$ group. Asterisks indicate the electrodes showing a significant power enhancement for the $14 \mathrm{~Hz}$ group compared to the $5.5 \mathrm{~Hz}$ group.

Having established that entrainment directly affected oscillatory activity during the retention interval, we next tested whether entrainment affected endogenous EEG activity during the subsequent memory test. If entrainment led to persistent changes in the excitability of the networks that generate theta activity, then there are at least two ways in which it could affect performance (Rugg \& Wilding, 2000; Wilding \& Ranganath, 2011; Mitchell \& Johnson, 2009). One possibility is that theta entrainment enhances recollective processes that are engaged specifically following recognition (i.e., "Post-retrieval Monitoring"). In this case, we would expect an interaction between theta entrainment and source recognition, such that theta power in the $5.5 \mathrm{~Hz}$ group would be elevated specifically on Item+Source trials (cf. Rugg \& Wilding, 2000). Alternatively, $5.5 \mathrm{~Hz}$ entrainment could increase the engagement of memory monitoring and control processes (i.e., "retrieval orientation") that are engaged prior to successful memory retrieval (Johnson, Kounios, \& Nolde, 1997; Ranganath \& Paller, 1999, 2000; Johnson \& Rugg, 2006), in which case we would expect to see a main effect of entrainment group, such that theta power during retrieval would be generally enhanced following $5.5 \mathrm{~Hz}$ entrainment.

We first compared theta activity between the $5.5 \mathrm{~Hz}$ entrainment group and the $14 \mathrm{~Hz}$ control group for all trial types. Statistical analysis showed sustained theta activity increases over frontal electrodes for the $5.5 \mathrm{~Hz}$ entrainment group compared to the $14 \mathrm{~Hz}$ control group (0.25-1 sec, cluster $p=0.0392$; Figure $4 A$ ). Collapsing over time, theta increases were most pronounced over frontal and parietal electrodes (Figure 4B). These effects were apparent only below $7 \mathrm{~Hz}$, and present in both total theta activity (Figure 5A; Supp. Figure 1) and induced theta activity (Figure 5B; Supp. Figure 2). Although beta activity appeared to be numerically increased at right parietal 
electrodes (Figure 4C), there were no significant effects in the beta band. We next tested for selective increases for Item+Source trials compared to Item-Only trials. Although numerically larger, there were no significant differences in theta activity between the $5.5 \mathrm{~Hz}$ group and the $14 \mathrm{~Hz}$ group. Accordingly, the data are most consistent with an account by which theta entrainment modulated one's retrieval orientation in a manner that enhanced successful source monitoring (Rugg \& Wilding, 2000; Mitchell \& Johnson, 2009).

A
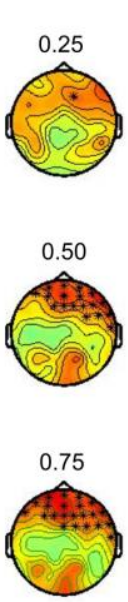

Theta power $(4-6 \mathrm{~Hz})$ : $5.5 \mathrm{~Hz}$ entrainment group - $14 \mathrm{~Hz}$ entrainment group

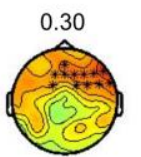

0.55
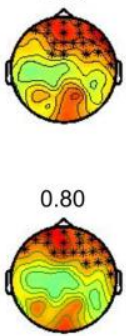
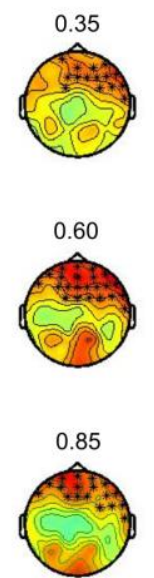
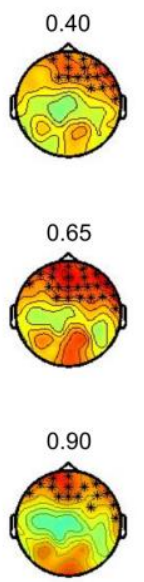
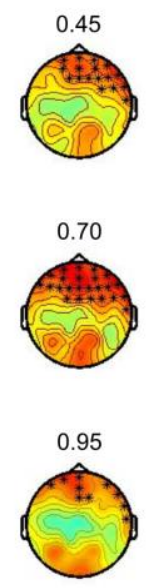

Theta power $(4-6 \mathrm{~Hz})$

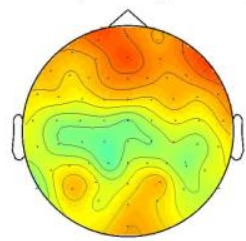

C
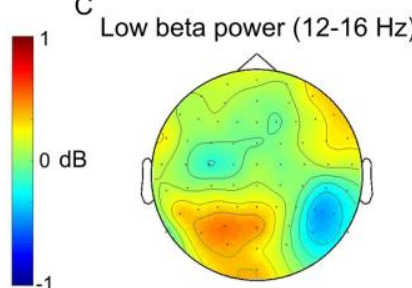

Figure 4: Theta entrainment enhances spontaneous theta power during memory retrieval. A) Topographies showing enhanced theta activity for the $5.5 \mathrm{~Hz}$ entrainment group compared to the $14 \mathrm{~Hz}$ control group. Asterisks show electrodes in significant cluster over frontal electrodes. B) Enhanced theta activity averaged across time for the $5.5 \mathrm{~Hz}$ entrainment group compared to the $14 \mathrm{~Hz}$ control group. C) Low beta activity shows no significant differences between the two groups.
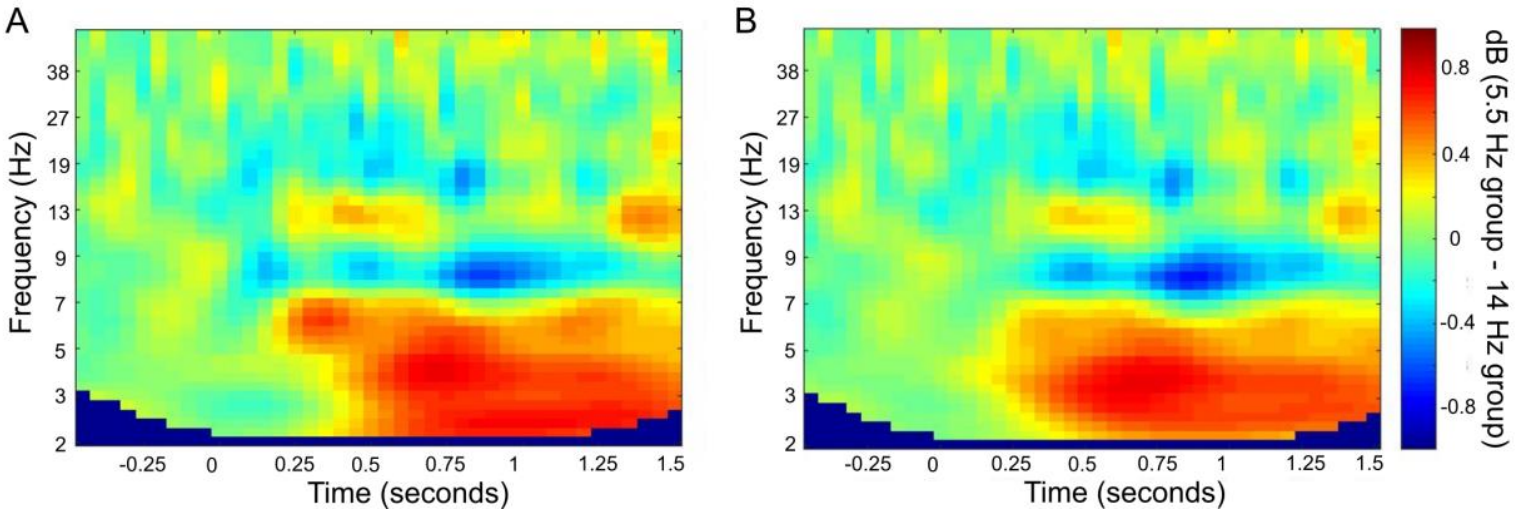

Figure 5: Time frequency representations showing differences between the $5.5 \mathrm{~Hz}$ entrainment group and the $14 \mathrm{~Hz}$ control group over frontal electrodes. Specific increased power is seen below $\sim \mathrm{Hz}$ in both an analysis of, A) total power, and B) induced power. 


\section{Discussion:}

To summarize, results from two independent experiments showed that entrainment of theta oscillations enhanced source memory performance, when compared against white noise (Expt. 1) or entrainment at a non-theta frequency (Expt. 2). Furthermore, entrainment at theta frequency increased spontaneous theta activity during memory retrieval, suggesting that it had lasting effects on the cortico-hippocampal networks that generate theta activity. The results demonstrate that direct manipulations of theta oscillations have relatively selective effects on memory retrieval and endogenous neural activity. These studies indicate that theta oscillations reflect neural processes that directly contribute to successful episodic memory retrieval.

Previous attempts to influence neural oscillatory activity with entrainment have largely focused on either short periods of flickering visual stimulation (Williams, 2001; Wimber et al., 2012; Sato, 2013) or auditory tones (Will and Berg, 2007; Henry and Obleser, 2012; Herrmann et al., 2013a; Henry et al., 2014; Ronconi et al., 2016) on a trial-by-trial basis during encoding. For instance, Williams (2001) reported that flicker stimulation at $10 \mathrm{~Hz}$ (alpha frequency) enhanced memory encoding. Wimber et al. (2012) used flickering visual stimuli presented at 6 or $10 \mathrm{~Hz}$ to entrain oscillations during encoding of words. This study did not report significant effects of entrainment frequency on memory performance, but EEG power at the flicker frequency was enhanced during memory retrieval. Another study found that flicker at $7.08 \mathrm{~Hz}$ induced transient changes in theta activity during spatial working memory encoding, but no cognitive effects were found for working memory performance (Sato, 2013).

In the present study, extended AVE was presented during the interval between memory encoding and retrieval in a between-subjects design. We chose this design because we expected that AVE during the retention interval would have lasting effects by enhancing the likelihood of theta states during the subsequent memory retrieval test. Although this hypothesis was speculative, the results of Experiment 2 demonstrated that $5.5 \mathrm{~Hz}$ entrainment increased theta power during the subsequent memory retrieval test. The results provide new evidence to demonstrate that prolonged theta entrainment can have lasting effects on subsequent neurophysiology and memory performance.

As noted earlier, the spectral characteristics of the AVE signals from the entrainment device were not constant throughout the entrainment period. The signals were delivered at variable frequencies during the first 15 minutes, and stabilized at $5.5 \mathrm{~Hz}$ from 15 to 30 minutes into the entrainment session. It is conceivable that the variations in entrainment frequencies in the first 15 minutes could contribute to the group differences in memory performance that we observed. However, this explanation does not account for the EEG data, which demonstrated a selective increase in power at $5.5 \mathrm{~Hz}$ for the theta entrainment group during the postentrainment memory retrieval task

Persistent effects of oscillatory entrainment have been demonstrated using other methods, such as non-invasive brain stimulation (for review see Veniero et al., 2015). Notably, direct 
effects of stimulation effects on evoked oscillatory power typically do not persist long beyond the stimulation period (Hanslmayr et al., 2014). Thus, it is more likely that persistent effects of stimulation, and possibly entrainment, are driven by other factors, such as NMDA receptor dependent plasticity (Nitsche et al., 2003; 2004) in prefrontal and parietal (Hsieh and Ranganath, 2014) circuits that naturally resonate at the stimulation frequency (Zaehle et al., 2010; Veniero et al., 2015; Vossen et al., 2015).

The present results help to clarify the role of theta activity in episodic memory. Many studies have reported that theta activity is enhanced during successful episodic memory retrieval (e.g., Guderian \& Duzel, 2005; Gruber et al., 2008; Addante et al., 2011, 2012; Meyer et al., 2015; Herweg et al., 2016; Backus et al., 2016; Clarke, Roberts and Ranganath, 2018; Crivelli-Decker et al., 2018), but the functional significance of these enhancements is unclear. Our EEG analyses provide some insights into the cognitive processes that were impacted by theta entrainment. The source memory task used here requires both recollection of past events (Yonelinas, 2002) and the accurate monitoring and evaluation of recollected details (Mitchell \& Johnson, 2009; Johnson et al., 2012). Because we did not find a selective effect of entrainment on source memory, the results do not provide evidence for the idea that theta activity solely enhanced memory consolidation by stimulating reactivation of studied information (Fuentemilla et al., 2010) or that it enhanced recollective processes such as post-retrieval monitoring (Rugg and Wilding, 2000; Mitchell \& Johnson, 2009). In the absence of such a selective effect, the overall enhancement of theta power in the $5.5 \mathrm{~Hz}$ entrainment group is more consistent with enhancement of processes that generally enhance source monitoring. For instance, entrainment could have led participants to change their "retrieval orientation" (Rugg \& Wilding, 2000) and selectively attend to retrieved details that are particularly diagnostic to successful source discrimination (Ranganath \& Paller, 1999; 2000; Mitchell \& Johnson, 2009; Wilding \& Ranganath, 2011). It is also possible that $5.5 \mathrm{~Hz}$ entrainment enhanced the ability to engage in a different kind of preparatory state that enhances the likelihood of recollection ("retrieval mode"; cf. Lepage et al., 2000). Both of these concepts can account for the selective effect of theta entrainment on source memory accuracy and the overall effect of entrainment on theta activity during memory retrieval.

The present results are somewhat discrepant with findings from a recent study that investigated the effects of 5 sessions of transcranial magnetic stimulation (TMS) to parietal cortex (Nilakantan et al., 2017). Following up on work by Wang, Voss, and colleagues (Wang et al., 2014, 2015), Nilakantan et al. targeted stimulation to parietal regions that show high functional connectivity with the hippocampus. Wang et al. $(2014,2015)$ previously showed that, following the stimulation sessions, those who received parietal stimulation showed better performance on an episodic memory test and increased intrinsic functional connectivity within a posterior medial cortico-hippocampal network (Libby et al., 2012; Ranganath \& Ritchey, 2012) that includes potential sources of scalp-recorded theta activity in humans (Hsieh and Ranganath, 2014). Using a similar procedure, but a different memory paradigm, Nilakantan et al. found that stimulation did not increase the likelihood of recollection, but it increased the precision of 
recollected memories. Surprisingly, participants in the stimulation group showed a reduction in theta activity during memory retrieval. Although it is surprising that their manipulation reduced theta activity and enhanced the precision of recollection, there are many possible explanations for their results. One possibility is that their reported enhancement in recollection was driven by effects other than theta, such as enhancement of sub-delta $(<1 \mathrm{~Hz})$ oscillations that could mediate intrinsic functional connectivity measured by fMRI (He et al., 2008). Another, nonexclusive, possibility is that theta activity promotes engagement of cognitive control processes. If parietal TMS enhanced hippocampal function, as suggested by Wang et al., $(2014,2015)$, then this might have enhanced spontaneous recollection and reduced the need for cognitive control during retrieval. This account is totally speculative, but it can potentially explain the reduction in theta activity following stimulation in Nilakantan et al. (2017), along with the general finding that theta activity is associated with improved source monitoring (Mitchell and Johnson, 2009).

One potential implication of the present results is that enhancement of oscillatory activity might be a promising target for improving learning and memory performance. As a noninvasive approach that does not require the administration of electrical current or strong magnetic fields, AVE could be promising for patients with memory disorders that are characterized by abnormal oscillatory patterns (Voytek and Knight, 2015). Further research is needed, however, to test the generalizability of the reported effects to other oscillatory entrainment methods and to other memory paradigms. Also, it will be important to test whether repeated theta entrainment could elicit lasting effects on episodic memory and endogenous theta activity in healthy individuals and in clinical populations.

\section{References}

Addante RJ, Watrous AJ, Yonelinas AP, Ekstrom AD, Ranganath C (2011) Prestimulus theta activity predicts correct source memory retrieval. Proc Natl Acad Sci U S A 108:1070210707. doi: 10710.11073/pnas.1014528108. Epub 1014522011 Jun 1014528113.

Addante RJ, Ranganath C, Yonelinas AP (2012) Examining ERP correlates of recognition memory: Evidence of accurate source recognition without recollection. Neuroimage, 62:1, 439450 .

Anderson MC, Hanslmayr S (2014) Neural mechanisms of motivated forgetting. Trends Cogn Sci 18:279-292. doi: 210.1016/j.tics.2014.1003.1002. Epub 2014 Apr 1018.

Asada H, Fukuda Y, Tsunoda S, Yamaguchi M, Tonoike M (1999) Frontal midline theta rhythms reflect alternative activation of prefrontal cortex and anterior cingulate cortex in humans. Neurosci Lett 274:29-32. 
Backus AR, Schoffelen JM, Szebényi S, Hanslmayr S, Doeller CF (2016) Hippocampal-prefrontal theta oscillations support memory integration. Current Biology, 26(4), 450-457.

Calderone DJ, Lakatos P, Butler PD, Castellanos FX (2014) Entrainment of neural oscillations as a modifiable substrate of attention. Trends Cogn Sci 18:300-309. doi: 310.1016/j.tics.2014.1002.1005. Epub 2014 Mar 1012.

Chaumon M, Bishop DV, Busch NA (2015) A practical guide to the selection of independent components of the electroencephalogram for artifact correction. J Neurosci Methods 250:47-63.:10.1016/j.jneumeth.2015.1002.1025. Epub 2015 Mar 1016.

Chun MM, Johnson MK (2011) Memory: enduring traces of perceptual and reflective attention. Neuron 72:520-535. doi: 510.1016/j.neuron.2011.1010.1026.

Clarke, A., Roberts, B. M., \& Ranganath, C. (2018). Neural oscillations during conditional associative learning. Neurolmage, 174, 485-493.

Crivelli-Decker, J., Hsieh, L. T., Clarke, A., \& Ranganath, C. (2018). Theta oscillations promote temporal sequence learning. Neurobiology of learning and memory.

Delorme A, Makeig S (2004) EEGLAB: an open source toolbox for analysis of single-trial EEG dynamics including independent component analysis. J Neurosci Methods 134:9-21.

Delorme A, Sejnowski T, Makeig S (2007) Enhanced detection of artifacts in EEG data using higher-order statistics and independent component analysis. Neuroimage 34:14431449. Epub 2006 Dec 1426.

Foster BL, Kaveh A, Dastjerdi M, Miller KJ, Parvizi J (2013) Human retrosplenial cortex displays transient theta phase locking with medial temporal cortex prior to activation during autobiographical memory retrieval. J Neurosci 33:10439-10446. doi: 10410.11523/JNEUROSCI.10513-10413.12013.

Foster BL, Parvizi J (2012) Resting oscillations and cross-frequency coupling in the human posteromedial cortex. Neuroimage 60:384-391. doi: 310.1016/j.neuroimage.2011.1012.1019. Epub 2011 Dec 1029.

Fuentemilla L, Barnes GR, Duzel E, Levine B (2014) Theta oscillations orchestrate medial temporal lobe and neocortex in remembering autobiographical memories. Neuroimage 85:730-737. doi: 710.1016/j.neuroimage.2013.1008.1029. Epub 2013 Aug 1023.

Fuentemilla L, Penny WD, Cashdollar N, Bunzeck N, Duzel E (2010) Theta-coupled periodic replay in working memory. Curr Biol 20:606-612. doi: 610.1016/j.cub.2010.1001.1057. Epub 2010 Mar 1018. 
Gruber T, Tsivilis D, Giabbiconi CM, Müller MM (2008) Induced electroencephalogram oscillations during source memory: familiarity is reflected in the gamma band, recollection in the theta band. Journal of cognitive neuroscience, 20:6, 1043-1053.

Guderian, S. and Düzel, E. (2005), Induced theta oscillations mediate large-scale synchrony with mediotemporal areas during recollection in humans. Hippocampus, 15: 901-912. doi:10.1002/hipo.20125

Haenschel C, Bittner RA, Waltz J, Haertling F, Wibral M, Singer W, Linden DE, Rodriguez E (2009) Cortical oscillatory activity is critical for working memory as revealed by deficits in earlyonset schizophrenia. J Neurosci 29:9481-9489. doi: 9410.1523/JNEUROSCI.14289409.2009.

Halbleib A, Gratkowski M, Schwab K, Ligges C, Witte H, Haueisen J (2012) Topographic analysis of engagement and disengagement of neural oscillators in photic driving: a combined electroencephalogram/magnetoencephalogram study. J Clin Neurophysiol 29:33-41. doi: 10.1097/WNP.1090b1013e318246ad318246e.

Hanslmayr S, Matuschek J, Fellner MC (2014) Entrainment of prefrontal beta oscillations induces an endogenous echo and impairs memory formation. Curr Biol 24:904-909. doi: 910.1016/j.cub.2014.1003.1007. Epub 2014 Mar 1027.

Hanslmayr S, Staudigl T, Aslan A, Bauml KH (2010) Theta oscillations predict the detrimental effects of memory retrieval. Cogn Affect Behav Neurosci 10:329-338. doi: 310.3758/CABN.3710.3753.3329.

Hasselmo ME, Hay J, Ilyn M, Gorchetchnikov A (2002) Neuromodulation, theta rhythm and rat spatial navigation. Neural Netw 15:689-707.

Henry MJ, Obleser J (2012) Frequency modulation entrains slow neural oscillations and optimizes human listening behavior. Proc Natl Acad Sci U S A 109:20095-20100. doi: 20010.21073/pnas.1213390109. Epub 1213392012 Nov 1213390114.

Henry MJ, Herrmann B, Obleser J (2014) Entrained neural oscillations in multiple frequency bands comodulate behavior. Proc Natl Acad Sci U S A 111:14935-14940. doi: 14910.11073/pnas.1408741111. Epub 1408742014 Sep 1408741129.

Herrmann B, Henry MJ, Grigutsch M, Obleser J (2013a) Oscillatory phase dynamics in neural entrainment underpin illusory percepts of time. J Neurosci 33:15799-15809. doi: 15710.11523/JNEUROSCI.11434-15713.12013. 
Herrmann CS, Rach S, Neuling T, Struber D (2013b) Transcranial alternating current stimulation: a review of the underlying mechanisms and modulation of cognitive processes. Front Hum Neurosci 7:279.:10.3389/fnhum.2013.00279. eCollection 02013.

Herrmann CS, Struber D, Helfrich RF, Engel AK (2015) EEG oscillations: From correlation to causality. Int J Psychophysiol 4:00033-00031.

Herweg NA, Apitz T, Leicht G, Mulert C, Fuentemilla L, Bunzeck N (2016) Theta-Alpha Oscillations Bind the Hippocampus, Prefrontal Cortex, and Striatum during Recollection: Evidence from Simultaneous EEG-fMRI. Journal of Neuroscience, 36:12, 3579-3587.

Hsieh LT, Ranganath C (2014) Frontal midline theta oscillations during working memory maintenance and episodic encoding and retrieval. Neuroimage 85:721-729. doi: 710.1016/j.neuroimage.2013.1008.1003. Epub 2013 Aug 1018.

Ishii R, Shinosaki K, Ukai S, Inouye T, Ishihara T, Yoshimine T, Hirabuki N, Asada H, Kihara T, Robinson SE, Takeda M (1999) Medial prefrontal cortex generates frontal midline theta rhythm. Neuroreport 10:675-679.

Jacobs J, Hwang G, Curran T, Kahana MJ (2006) EEG oscillations and recognition memory: theta correlates of memory retrieval and decision making. Neuroimage 32:978-987. Epub $2006 \mathrm{Jul} 2012$.

Johnson MK, Kounios J, Nolde SF (1997). Electrophysiological brain activity and memory source monitoring. NeuroReport, 8(5), 1317-1320.

Johnson MK, Hashtroudi S, Lindsay DS (1993) Source monitoring. Psychol Bull 114:3-28.

Johnson MK, Raye CL, Mitchell KJ, Ankudowich E (2012) The cognitive neuroscience of true and false memories. Nebr Symp Motiv 58:15-52.

Johnson JD, Rugg MD (2006). Electrophysiological correlates of retrieval processing: effects of consistent versus inconsistent retrieval demands. Journal of cognitive neuroscience, 18(9), 1531-1544.

Jung TP, Makeig S, Westerfield M, Townsend J, Courchesne E, Sejnowski TJ (2000a) Removal of eye activity artifacts from visual event-related potentials in normal and clinical subjects. Clin Neurophysiol 111:1745-1758.

Jung TP, Makeig S, Humphries C, Lee TW, McKeown MJ, Iragui V, Sejnowski TJ (2000b) Removing electroencephalographic artifacts by blind source separation. Psychophysiology 37:163178. 
Kahn I, Andrews-Hanna JR, Vincent JL, Snyder AZ, Buckner RL (2008) Distinct cortical anatomy linked to subregions of the medial temporal lobe revealed by intrinsic functional connectivity. J Neurophysiol 100:129-139. doi: 110.1152/jn.00077.02008. Epub 02008 Apr 00072.

Klem GH, Luders HO, Jasper HH, Elger C (1999) The ten-twenty electrode system of the International Federation. The International Federation of Clinical Neurophysiology. Electroencephalogr Clin Neurophysiol Suppl 52:3-6.

Klimesch W, Doppelmayr M, Schimke H, Ripper B (1997) Theta synchronization and alpha desynchronization in a memory task. Psychophysiology 34:169-176.

Lakatos P, Karmos G, Mehta AD, Ulbert I, Schroeder CE (2008) Entrainment of neuronal oscillations as a mechanism of attentional selection. Science 320:110-113. doi: $110.1126 /$ science.1154735.

Landfield PW, McGaugh JL, Tusa RJ (1972) Theta rhythm: a temporal correlate of memory storage processes in the rat. Science 175:87-89.

Lepage M, Ghaffar O, Nyberg L, Tulving E (2000) Prefrontal cortex and episodic memory retrieval mode. Proc Natl Acad Sci U S A 97:506-511.

Libby LA, Ekstrom AD, Ragland JD, Ranganath C (2012) Differential connectivity of perirhinal and parahippocampal cortices within human hippocampal subregions revealed by highresolution functional imaging. J Neurosci 32:6550-6560. doi: 6510.1523/JNEUROSCI.3711-6511.2012.

Maris, E., \& Oostenveld, R. (2007). Nonparametric statistical testing of EEG-and MEGdata. Journal of neuroscience methods, 164(1), 177-190.

Meyer L, Grigutsch M, Schmuck N, Gaston P, Friederici AD (2015) Frontal-posterior theta oscillations reflect memory retrieval during sentence comprehension. Cortex, 71, 205218.

Mickes L, Wais PE, Wixted JT (2009) Recollection Is a Continuous Process: Implications for DualProcess Theories of Recognition Memory. Psychol Sci 20:4, 509-515.

Mitchell KJ, Johnson MK (2009) Source monitoring 15 years later: what have we learned from fMRI about the neural mechanisms of source memory? Psychol Bull 135:638-677. doi: $610.1037 / \mathrm{a} 0015849$. 
Nitsche MA, Frick K, Henschke U, Schlitterlau A, Liebetanz D, Lang N, Paulus W (2003). Pharmacological modulation of cortical excitability shifts induced by transcranial direct current stimulation in humans. The Journal of physiology, 553(1), 293-301.

Nitsche MA, Grundey J, Liebetanz D, Lang N, Tergau F, Paulus W (2004). Catecholaminergic consolidation of motor cortical neuroplasticity in humans. Cerebral Cortex, 14(11), 12401245.

Nilakantan AS, Bridge DJ, Gagnon EP, VanHaerents SA, Voss JL (2017) Stimulation of the posterior cortical-hippocampal network enhances precision of memory recollection. Current Biology, 27(3), 465-470.

Oostenveld, R., Fries, P., Maris, E., \& Schoffelen, J. M. (2011). FieldTrip: open source software for advanced analysis of MEG, EEG, and invasive electrophysiological data. Computational intelligence and neuroscience, 2011, 1.

Ranganath C, Paller KA (1999). Frontal brain potentials during recognition are modulated by requirements to retrieve perceptual detail. Neuron, 22(3), 605-613.

Ranganath C, Paller KA (2000). Neural correlates of memory retrieval and evaluation. Cognitive Brain Research, 9(2), 209-222.

Roach BJ, Mathalon DH (2008) Event-related EEG time-frequency analysis: an overview of measures and an analysis of early gamma band phase locking in schizophrenia. Schizophr Bull 34:907-926. doi: 910.1093/schbul/sbn1093. Epub 2008 Aug 1096.

Roediger HL (1996) Memory illusions. Journal of Memory and Language 35:76-100.

Ronconi L, Pincham HL, Cristoforetti G, Facoetti A, Szucs D (2016) Shaping prestimulus neural activity with auditory rhythmic stimulation improves the temporal allocation of attention. Neuroreport 27:487-494. doi: 410.1097/WNR.0000000000000565.

Rugg MD, Wilding EL (2000) Retrieval processing and episodic memory. Trends Cogn Sci 4:108115.

Rutishauser U, Ross IB, Mamelak AN, Schuman EM (2010) Human memory strength is predicted by theta-frequency phase-locking of single neurons. Nature 464:903-907. doi: 910.1038/nature08860. Epub 02010 Mar 08824.

Sato N (2013) Fast entrainment of human electroencephalogram to a theta-band photic flicker during successful memory encoding. Front Hum Neurosci 7:208.:10.3389/fnhum.2013.00208. eCollection 02013. 
Schacter DL, Norman KA, Koutstaal W (1998) The cognitive neuroscience of constructive memory. Annu Rev Psychol 49:289-318.

Staudigl T, Hanslmayr S (2013) Theta oscillations at encoding mediate the context-dependent nature of human episodic memory. Curr Biol 23:1101-1106. doi:

1110.1016/j.cub.2013.1104.1074. Epub 2013 Jun 1106.

Suthana N, Fried I (2014) Deep brain stimulation for enhancement of learning and memory. Neuroimage 85:996-1002. doi: 1010.1016/j.neuroimage.2013.1007.1066. Epub 2013 Aug 1003.

Teplan M, Krakovska A, Stolc S (2011) Direct effects of audio-visual stimulation on EEG. Comput Methods Programs Biomed 102:17-24. doi: 10.1016/j.cmpb.2010.1011.1013. Epub 2011 Jan 1022.

Thut G, Schyns PG, Gross J (2011) Entrainment of perceptually relevant brain oscillations by noninvasive rhythmic stimulation of the human brain. Front Psychol 2:170.:10.3389/fpsyg.2011.00170. eCollection 02011.

Veniero D, Vossen A, Gross J, Thut G (2015) Lasting EEG/MEG Aftereffects of Rhythmic Transcranial Brain Stimulation: Level of Control Over Oscillatory Network Activity. Front Cell Neurosci 9:477.:10.3389/fncel.2015.00477. eCollection 02015.

Vossen A, Gross J, Thut G (2015) Alpha Power Increase After Transcranial Alternating Current Stimulation at Alpha Frequency (alpha-tACS) Reflects Plastic Changes Rather Than Entrainment. Brain Stimul 8:499-508. doi: 410.1016/j.brs.2014.1012.1004. Epub 2014 Dec 1020.

Voytek B, Knight RT (2015) Dynamic network communication as a unifying neural basis for cognition, development, aging, and disease. Biol Psychiatry 77:1089-1097. doi: 1010.1016/j.biopsych.2015.1004.1016. Epub 2015 Apr 1028.

Walter WG (1953) The living brain. New York, NY: Norton.

Wang JX, Voss JL (2015) Long-lasting enhancements of memory and hippocampal-cortical functional connectivity following multiple-day targeted noninvasive stimulation. Hippocampus 25:877-883. doi: 810.1002/hipo.22416. Epub 22015 Feb 22411.

Wang JX, Rogers LM, Gross EZ, Ryals AJ, Dokucu ME, Brandstatt KL, Hermiller MS, Voss JL (2014) Targeted enhancement of cortical-hippocampal brain networks and associative memory. Science 345:1054-1057. doi: 1010.1126/science.1252900. 
Wang SF, Ritchey M, Libby LA, Ranganath C (2016) Functional connectivity based parcellation of the human medial temporal lobe. Neurobiol Learn Mem 19:00017-00014.

Wilding E, Ranganath C (2011) Electrophysiological correlates of episodic memory processes. Oxford handbook of ERP components, Oxford University Press. Pp 373-396.

Will U, Berg E (2007) Brain wave synchronization and entrainment to periodic acoustic stimuli. Neurosci Lett 424:55-60. Epub 2007 Aug 2006.

Williams JH (2001) Frequency-specific effects of flicker on recognition memory. Neuroscience 104:283-286.

Wimber M, Maass A, Staudigl T, Richardson-Klavehn A, Hanslmayr S (2012) Rapid memory reactivation revealed by oscillatory entrainment. Curr Biol 22:1482-1486. doi: 1410.1016/j.cub.2012.1405.1054. Epub 2012 Jul 1412.

Winson J (1978) Loss of hippocampal theta rhythm results in spatial memory deficit in the rat. Science 201:160-163.

Yartsev MM, Witter MP, Ulanovsky N (2011) Grid cells without theta oscillations in the entorhinal cortex of bats. Nature 479:103-107. doi: 110.1038/nature10583.

Yonelinas AP (2002) The Nature of Recollection and Familiarity: A review of 30 Years of Research. Journal of Memory and Language 46:441-517.

Yonelinas AP, Jacoby LL (2012) The process-dissociation approach two decades later: Convergence, boundary conditions, and new directions. Memory \& Cognition 40:5, 663 680.

Zaehle T, Rach S, Herrmann CS (2010) Transcranial alternating current stimulation enhances individual alpha activity in human EEG. PLoS One 5:e13766. doi:

13710.11371/journal.pone.0013766. 
Table 1: Mean performance (standard error of the mean) for the entrainment and control groups for both experiments

\begin{tabular}{llccccc} 
& \multicolumn{2}{c}{$\begin{array}{c}\text { Item+Source } \\
\text { hits }\end{array}$} & $\begin{array}{c}\text { Item Only } \\
\text { hits }\end{array}$ & False Alarms & Item d' & C \\
\hline Experiment 1 & Theta & $73.8 \%(2.1)$ & $11.2 \%(1.8)$ & $16.7 \%(1.9)$ & $1.94(0.11)$ & $-0.24(0.06)$ \\
& Control & $68.3 \%(1.5)$ & $12.2 \%(2.0)$ & $18.8 \%(2.3)$ & $1.73(0.07)$ & $-0.31(0.05)$ \\
Experiment 2 & Theta & $70.9 \%(1.3)$ & $11.9 \%(1.7)$ & $19.1 \%(3.1)$ & $1.96(0.14)$ & $-0.23(0.04)$ \\
& Control & $66.2 \%(1.9)$ & $16.2 \%(1.9)$ & $25.8 \%(2.7)$ & $1.65(0.10)$ & $-022(0.04)$
\end{tabular}

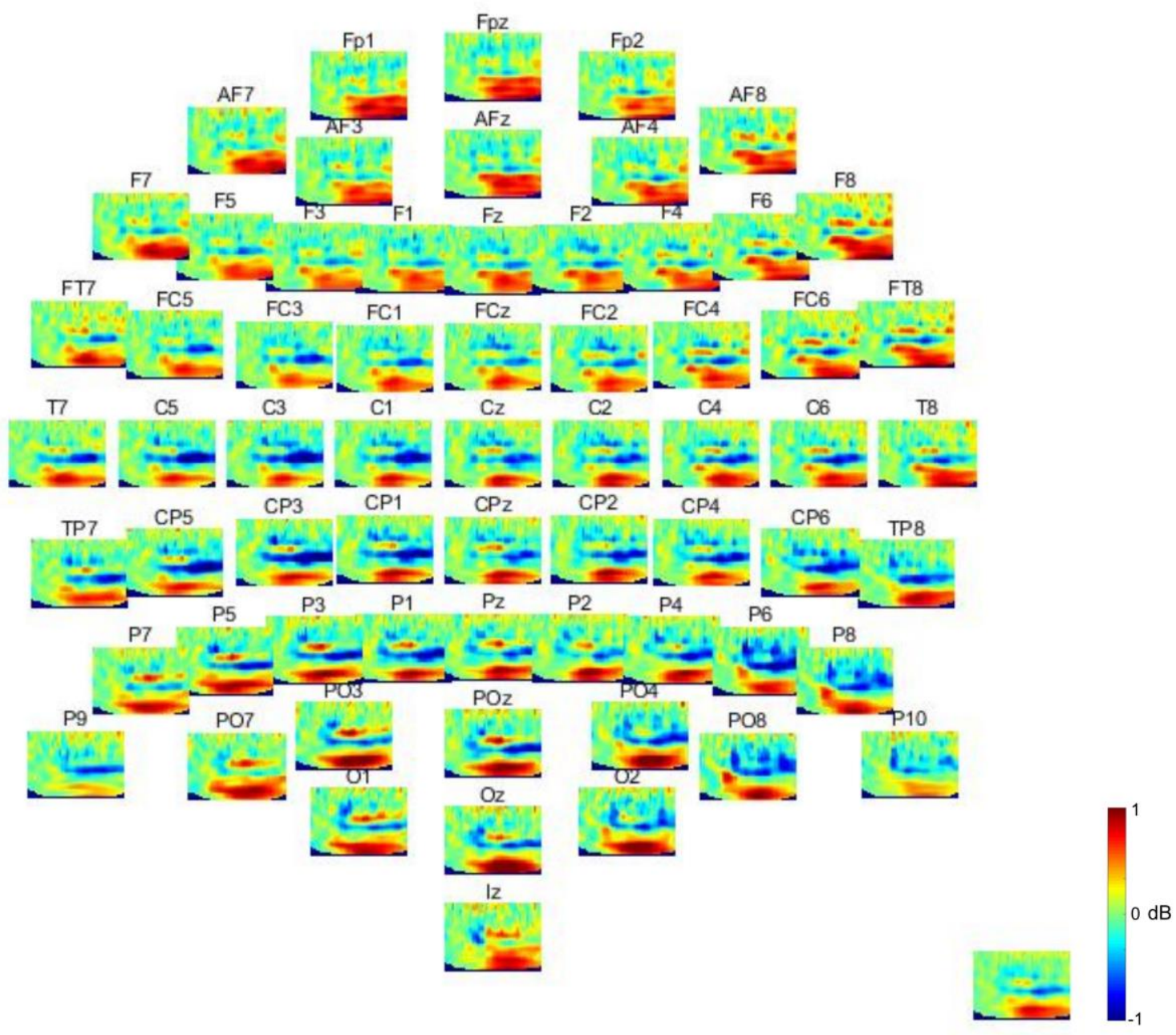

Sup Figure 1: Time frequency representations of total power showing differences between the $5.5 \mathrm{~Hz}$ entrainment group and the $14 \mathrm{~Hz}$ control group at each electrode between 2 and $50 \mathrm{~Hz}$. Data is combined across Item+Souce and Item only trials. 


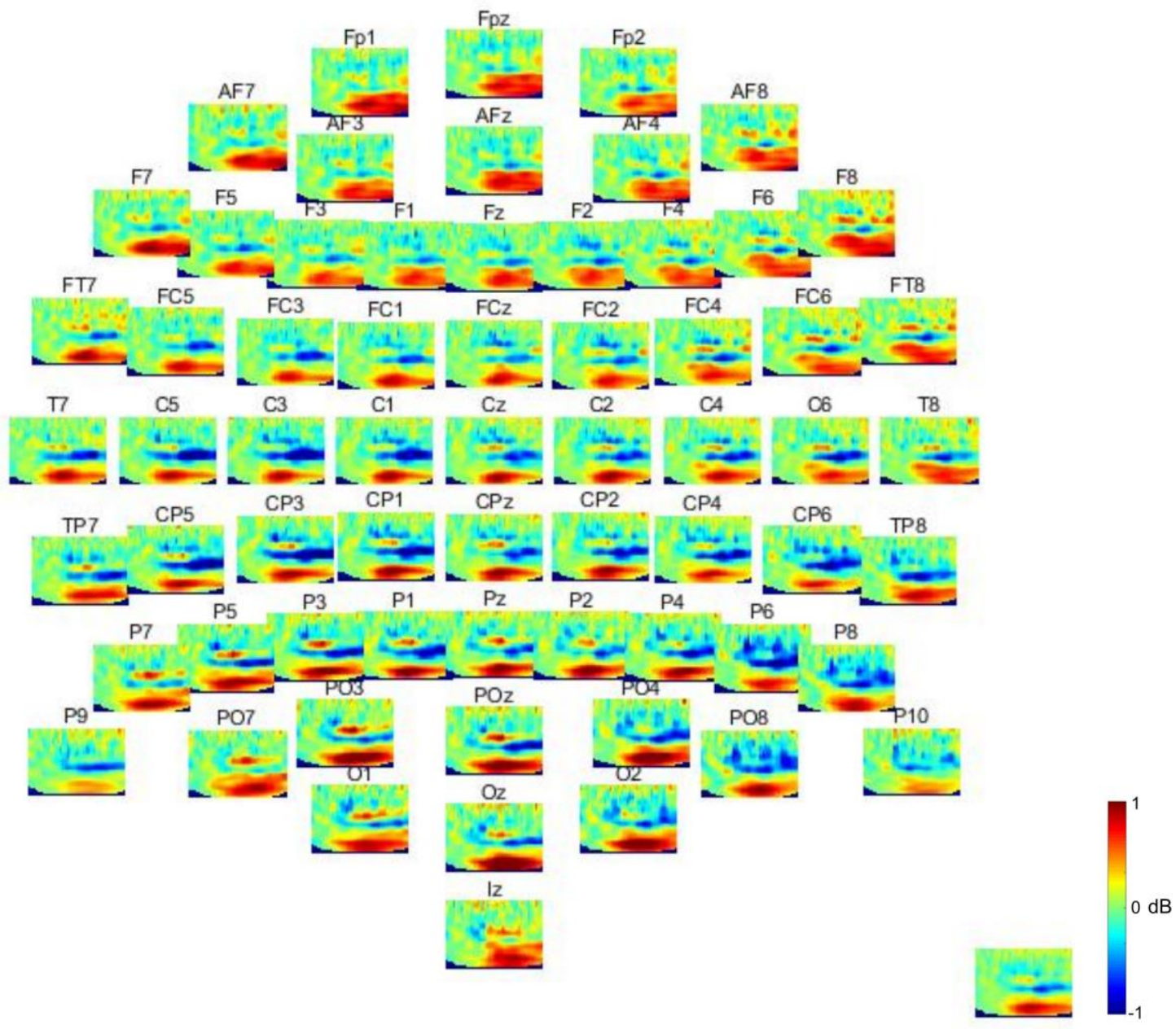

Sup Figure 2: Time frequency representations of induced power showing differences between the $5.5 \mathrm{~Hz}$ entrainment group and the $14 \mathrm{~Hz}$ control group at each electrode between 2 and $50 \mathrm{~Hz}$. Data is combined across Item+Souce and Item only trials. 